\title{
Gestatten, unser Name ist JADE
}

\author{
Künftige und frisch gebackene Allgemeinmediziner haben ein bundesweites Netzwerk gegründet. \\ Sie unterstützen sich beim Start ins Berufsleben - und mischen in der Berufspolitik mit.
}

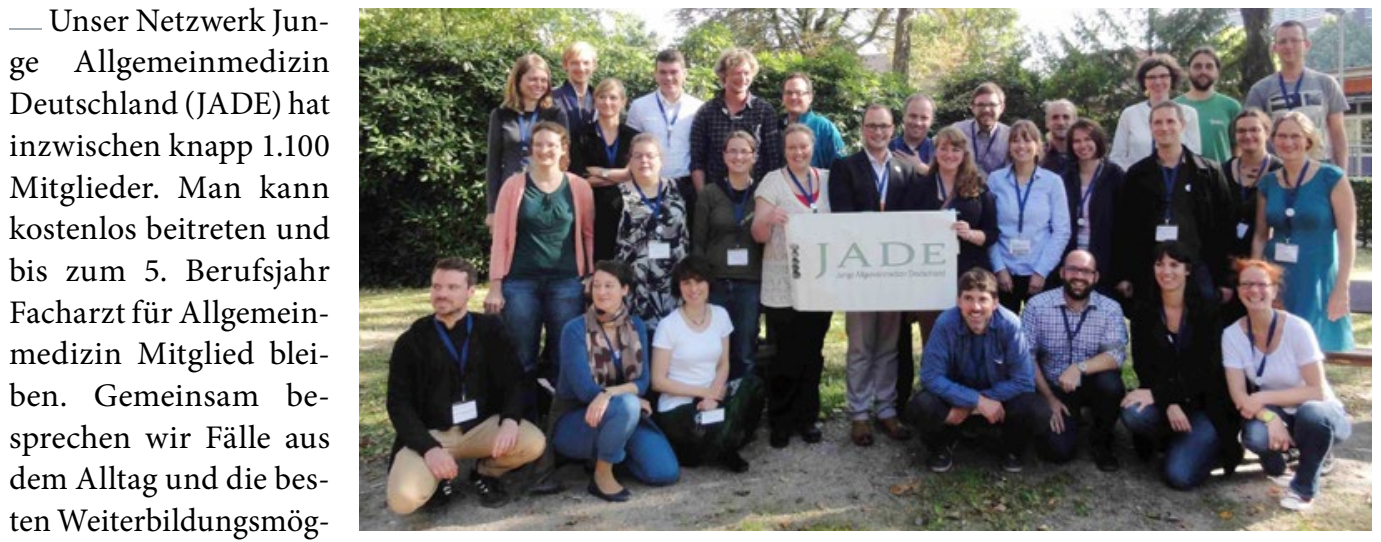

lichkeiten, wir tauschen Gut gelaunter Blick nach vorn: Vertreter des JADE-Netzwerks.

Tipps für kommende

Stellen aus und diskutieren Politisches rund um unser Fach. Dafür haben wir ein Online-Forum auf unserer Website www.jungeallgemeinmedizin.de.

Wir möchten Weiterbildungs-, Arbeits- und Forschungsbedingungen für junge Ärzte in der Allgemeinmedizin verbessern und uns gegenseitig bei Fragen zu unserem Berufsweg zur Seite stehen. Die JADE betrachten wir als zentralen Ansprechpartner auf dem Weg oder zu Beginn des Berufes Facharzt für Allgemeinmedizin - und als Möglichkeit, uns berufspolitisch einzubringen. Das Netzwerk lebt von den engagierten Diskussionen. Öffentliche Positionen stimmen wir basisdemokratisch ab.

Jeden Frühling gibt es ein JADE-Treffen in wechselnden Städten. Dort können sich Mitglieder aus allen Bundesländer austauschen, vernetzen und fortbilden. Zum Kongress der Deutschen Ge- sellschaft für Allgemeinmedizin und Familienmedizin (DEGAM) im Herbst treffen wir uns ein zweites Mal. Dort wählen wir jährlich auch die ehrenamtlich tätigen Vorstände der JADE, die sich für unsere Anliegen einsetzen.

\section{Vernetzt in ganz Europa}

Ergänzt wird dies durch die Öffentlichkeitsarbeit und die Vernetzung mit anderen jungen und zukünftigen Allgemeinmedizinern aus Europa und innerhalb Deutschlands. In den einzelnen Städten und Bundesländern treffen wir uns darüber hinaus in einer Vielzahl von Regionalgruppen und Stammtischen zur Fortbildung und zum lockeren Austausch.

Den Vorstand bilden zurzeit Dr. Marco Roos (Weiterbildung), Dr. Leonor Heinz (Netzwerk und Regionalgruppen), Dr. Hannah Haumann (Öffentlichkeits- arbeit, zurzeit kommissarische DEGAM-Kontaktperson), Dr. Christian Rechtenwald (Internationale Zusammenarbeit) und Dr. Jana Husemann (Junge Fachärzte).

Die JADE kooperiert mit den nationalen und internationalen Gesellschaften für Allgemeinmedizin, insbesondere mit der DEGAM, dem Hausärzteverband, dem Vasco da Gama Movement (VdGM) sowie der Weltorganisation für Allgemeinund Familienärzte (WONCA). Sie wahrt dabei ihre inhaltliche und organisatorische Eigenständigkeit. Wir sind unabhängig - auch von jeglichem Einfluss der Pharmaindustrie - und legen jede finanzielle Unterstützung offen.

Wir freuen uns darauf, in der MMW nun regelmäßig von uns zu berichten. Wir zeigen Ihnen die Welt der jungen Allgemeinmedizin, unsere spannendsten Arbeitsplätze und interessantesten medizinischen Fälle. Auch die aktuelle Gesundheitspolitik wollen wir uns vornehmen - denn natürlich haben wir eine recht genaue Vorstellung davon, wie wir die Zukunft die Allgemeinmedizin in Deutschland mitgestalten wollen.

- Catharina Escales

- AG Presse- und Öffentlichkeitsarbeit der JADE und Ärztin in Weiterbildung aus Hamburg 